\title{
Zetasizer Technique in Biochemistry
}

\section{Nagham Mahmood Aljamali*}

Department of Chemistry, College of Education, Kufa University, Iraq

*Corresponding author: Nagham Mahmood Aljamali, Department of Chemistry, College of Education, Kufa University, Iraq, Tel: +96433 340952; E-mail: dr.nagham_mj@yahoo.com

Rec date: Mar 20, 2015; Acc date: Mar 28, 2015; Pub date: April 06, 2015

Copyright: (C) 2015 Aljamali NM. This is an open-access article distributed under the terms of the Creative Commons Attribution License, which permits unrestricted use, distribution, and reproduction in any medium, provided the original author and source are credited.

\section{Abstract}

In this review study of zetasizer technique and, using in molecular weight of compounds and large molecules, applications in most of fields like bio molecules, determination of molecular weight of organic chemistry.

Keywords: Flexible; Nano; Coefficient; Physical

\section{Introduction}

Overview of Zetasizer: The SZ-100 nano partical series instruments are flexible analytical tools for characterizing the physical properties of small particles. Depending on the configuration and application the system can be used as a particle size analyzer, or also used to measure zeta potential, molecular weight, (MW) and second virial coefficient (A2). Typical applications for the SZ-100 include nano particles, colloids, emulsions, and submicron suspensions (Figure 1).

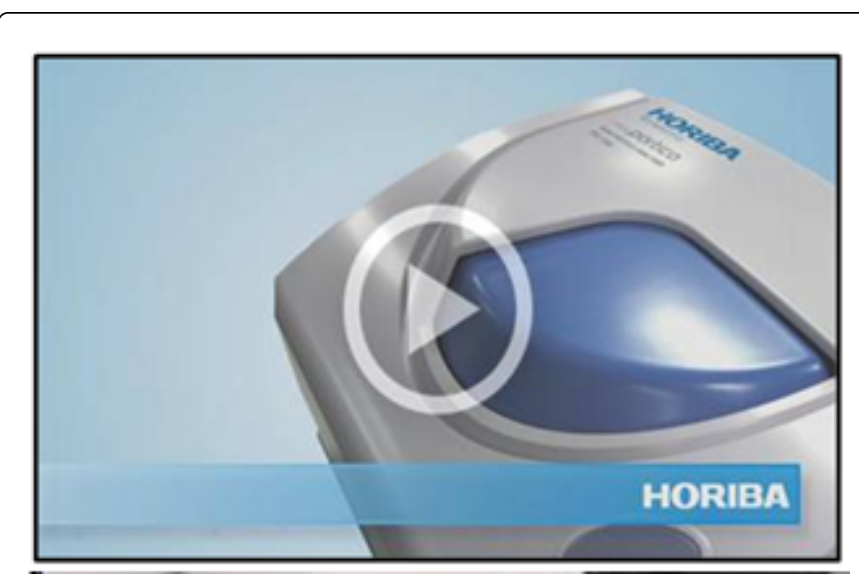

Figure 1: Zetasizer instrument.

Particle size analysis is performed by dynamic light scattering (DLS). Depending on the physical properties of the sample, the dynamic range is $0.3 \mathrm{~nm}-8 \mu \mathrm{m}$. The lower limit is influenced by concentration, how strongly the sample scatters light, and the presence of large, unwanted particles. The upper limit is influenced by the density of the sample since DLS is modeled on all motion coming from Brownian motion, not gravitational settling.

The charge on the surface of particles is characterized by the SZ-100 by measuring the zeta potential of a suspension. The sample is injected into a disposable cell and a measurement of the particle electrophoretic mobility results in the calculated zeta potential. The zeta potential of the sample is most often used as an indicator of dispersion stability. Large magnitude zeta potential values indicate that an electrostatically stabilized suspension will remain stable. The zeta potential is often measured as a function of $\mathrm{pH}$ or other change in the chemistry to help formulators create new products with a long shelf life. Conversely identifying conditions at which the zeta potential is zero (that is, the sample is at the isoelectric point) allows one to choose optimum conditions for flocculating and separating particles (Figure 2).

The same instrument can also be used to measure the molecular weight and second virial coefficient of proteins, polymers, and other molecules. The user prepares several solutions with known concentrations and then uses the system in a static light scattering mode to create a Debye plot, which results in a calculation of both MW and A2.

\section{Zetasizer}

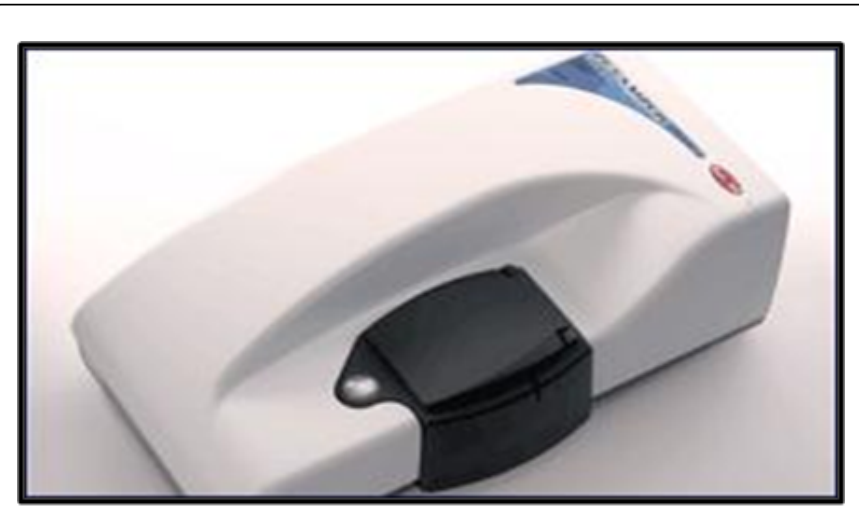

Figure 2: Zetasizer Nano.

\section{What does the Zetasizer Nano do?}

The Zetasizer Nano range of instruments provides the ability to measure three characteristics of particles or molecules in a liquid medium. These three fundamental parameters are particle size, zeta potential and molecular weight. By using the unique technology within the Zetasizer system, these parameters can be measured over a wide range of concentrations (Table 1). 
Page 2 of 5

\begin{tabular}{|l|l|l|l|}
\hline & Particle size and molecular size & Zeta potential (particle size diameter) & Molecular weight \\
\hline Measurement range & $0.3 \mathrm{~nm}-10.0$ microns (diameter) & $3.8 \mathrm{~nm}-100$ microns & $980 \mathrm{Da}-20 \mathrm{M} \mathrm{Da}$ \\
\hline Measurement principle & Dynamic Light Scattering & Electrophoretic Light Scattering & Static Light Scattering Using Debye Plot \\
\hline Sample volume range & $12-20 \mu \mathrm{L}$ & $150 \mu \mathrm{L}$ & $12-20 \mu \mathrm{L}$ \\
\hline Sample concentration & Up to $40 \% \mathrm{w} / \mathrm{v}$ & & \\
\hline Temperature Range & $0-120^{\circ} \mathrm{C}$ & \\
\hline
\end{tabular}

Table 1: Measurements of molecules.

\section{Features}

- Particle size, zeta potential, molecular weight, and second virial coefficient all in one instrument

- Wide range of particle sizes and concentrations

- Particles size measurements at both $90^{\circ}$ and $173^{\circ}$

- Multiple particle size measurement modes for working with small particles and weak scatterers

- Small volume cells for both particle size and zeta potential

- Zeta potential is a scientific term for electrokineticpotential [1] in colloidaldispersions. In the colloidal chemistry literature, it is usually denoted using the Greek letter zeta $(\zeta)$, hence $\zeta$-potential. From a theoretical viewpoint, the zeta potential is the electric potential in the interfacial double layer (DL) at the location of the slipping plane relative to a point in the bulk fluid away from the interface. In other words, zeta potential is the potential difference between the dispersion medium and the stationary layer of fluid attached to the dispersed particle.

- The zeta potential is caused by the net electrical charge contained within the region bounded by the slipping plane, and also depends on the location of that plane. Thus it is widely used for quantification of the magnitude of the charge. However, zeta potential is not equal to the Stern potential or electric surface potential in the double layer [2], because these are defined at different locations. Such assumptions of equality should be applied with caution. Nevertheless, zeta potential is often the only available path for characterization of double-layer properties.

- The zeta potential is a key indicator of the stability of colloidal dispersions. The magnitude of the zeta potential indicates the degree of electrostatic repulsion between adjacent, similarly charged particles in a dispersion. For molecules and particles that are small enough, a high zeta potential will confer stability, i.e., the solution or dispersion will resist aggregation. When the potential is small, attractive forces may exceed this repulsion and the dispersion may break and flocculate. So, colloids with high zeta potential (negative or positive) are electrically stabilized while colloids with low zeta potentials [3].

\section{Methods for Determining the Zeta Potential}

\section{Electrokinetic phenomena}

There are four electrochemical phenomena that can be used for the determination of zeta-potential, electrophoresis, electroosmosis, streaming and sedimentation potential. Electrophoresis and streaming potential are the most important commercial effects that have been exploited to determine zeta potential. The Malvern Zetasizer uses electrophoresis, however electroosmosis is an interfering effect that must be considered. There are a number of ways of accounting for this effect (Figures 3 and 4). The use of measurements at the stationary layer is one method that is discussed in the next section [4].

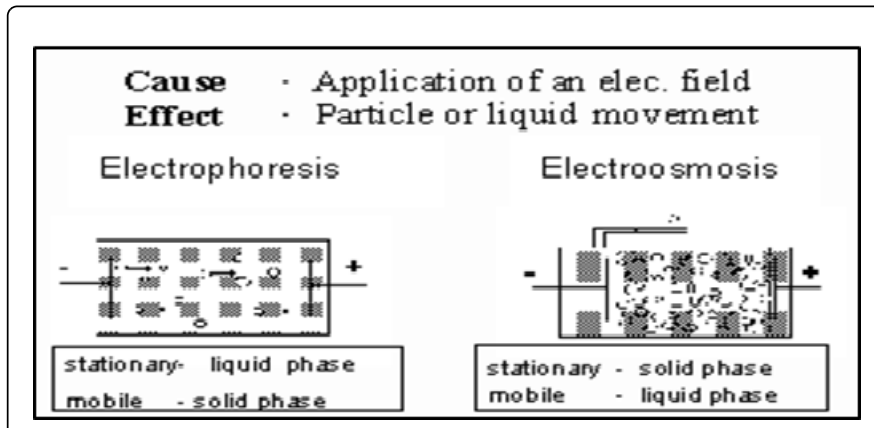

Figure 3: Basic principles of electrophoresis and electroosmosis.

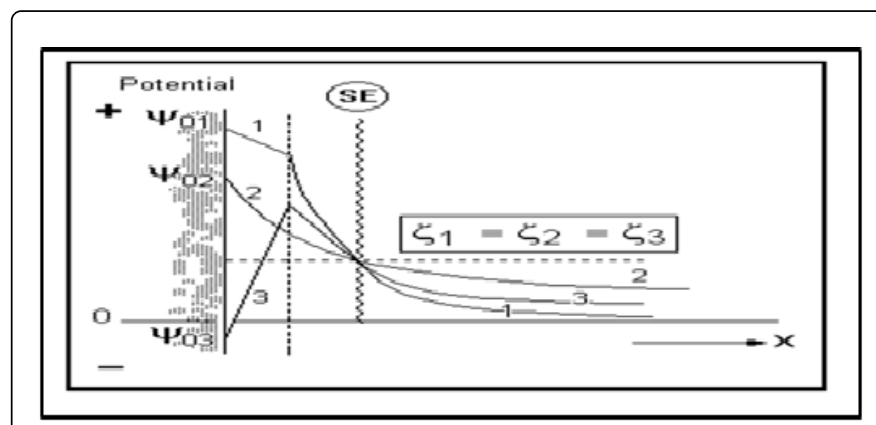

Figure 4: Potential trends at interfaces with differences in the chemical constitution, influence of specific ionic adsorption.

- Positively charged particles on the surface, moderate

- Adsorption of anions, moderate ionic strength positively charged particles with no specific adsorption, low concentration of electrolyte

- Negatively charged particles, strong specific cation adsorption, moderate electrolyte concentration [5].

\section{Micro-Electrophoresis}

The conventional method for measuring zeta potential is to observe the migration of particles in a dispersion with a microscope and 
Page 3 of 5

measure their velocity using a stopwatch. The current technique of laser Doppler velocimetry (LDV) has mostly replaced this laborious measurement procedure.

Both techniques are based on measurement of the migration rate of dispersed particles under the influence of an electric field. The observed velocity (v), divided by the strength of the applied electrical field $(\mathrm{E})$, is a direct measure of the electrophoretic mobility $(\mu \mathrm{E})$ of the particles examined $\mathrm{v} / \mathrm{E}=\mu \mathrm{E}$.

The zeta potential can be calculated from the mobility using the Henry equation.

Henry equation:

$$
\mu \mathrm{E}=\varepsilon^{\star} \xi^{\star} \mathrm{f}(\kappa \mathrm{a}) / 6 \pi \eta
$$

$\varepsilon$ - dielectric constant of the medium, $\eta$ - viscosity of the medium, $\mathrm{f}(\mathrm{\kappa a})$ - correction factor which takes into account the thickness of the double layer and particle diameter. The unit $\kappa$ is a reciprocal length. $1 / \kappa$ is frequently described as the thickness of the double layer. In practice an approximation can be made for $f(\kappa a)$, this is $f(\kappa a)=1.0$ for non-polar media, $f(\kappa \mathrm{a})=1.5$ for particle dispersions in polar media.

$\mathrm{f}(\kappa \mathrm{a})=1.5$ is a good approximation for particles $>100 \mathrm{~nm}$ in aqueous solutions with an ionic strength $>10-3 \mathrm{M}$. This is known as Smoluchowski approximation [6].

\section{Smoluchowski approximation}

$$
\mu \mathrm{E}=\varepsilon^{\star} \xi / 4 \pi \eta
$$

$$
\text { at } 25 \% \mathrm{C} \text { it is simplified to } \xi=12.85 \mu \mathrm{E} \mathrm{mV} \text {. }
$$

The second limiting case $f(\kappa a)=1.0$ applies for very small particles in media with a low permittivity. This is known as the Hückel approximation.

- If the conventional microscopic observation technique is compared with modern techniques, the advantages of the LDV methods are many. Some important advantages are:

- Statistically better measurements

- Seconds measurement time rather than 10-30 minutes

- Measurement of smaller particles, 5-10 $\mathrm{nm}$ rather than a minimum of $200 \mathrm{~nm}$

- Measurement of zeta potential distributions

- Improvement in measurement repeatability due to a reduction in the Joule heating effect

- Ability to automate complex measurements (e.g. $\mathrm{pH}$ or concentration dependences)

\section{The electrophoresis cell}

It comprises a quartz glass capillary $50 \mathrm{~mm}$ long, cross section 5 $\mathrm{mm}$ by $2 \mathrm{~mm}$, with electrodes at both ends. The capillary is sealed by valves to form a closed tube.

\section{The stationary layer}

In the same way that an electrical double layer is associated with charged particles, the same structure exists at the charged capillary wall. This leads to a liquid flow in the cell due to electroosmosis. This adds a velocity to the particle mobility at all points in the cell except at a special position called the stationary layer or stationary plane. This is created in a closed capillary because of the return flow in the center of the cell. At the stationary layer the fluid flow along the cell wall is exactly cancelled by the return flow. Measurements at this position give the true electrophoretic mobility (Figure 5) [7].

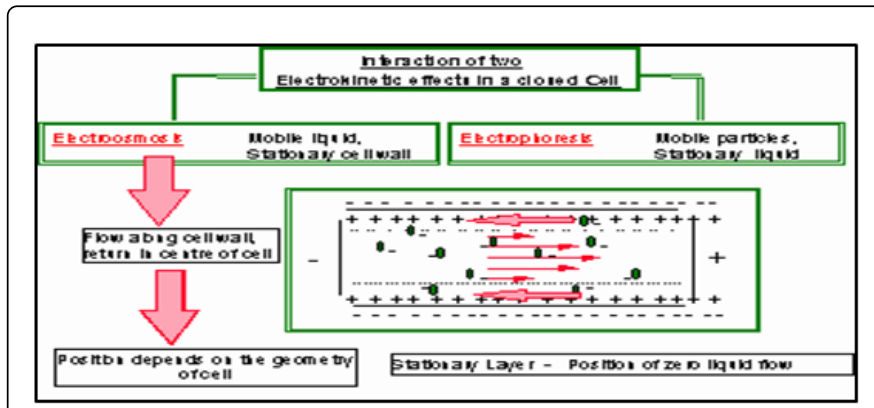

Figure 5: Electrokinetic effects in a closed electrophoresis cell.

The position of the stationary plane is dependent on the cell geometry and can be calculated for symmetrical shapes of capillary.

\section{Zeta potential measurement technique}

In contrast to the traditional microscope measuring technique, in the LDV method the particle image is replaced by the illumination of particles by intersecting laser beams, the observer's eye by the photomultiplier, and the stopwatch by the correlator [8].

The Malvern system is called the Zetasizer. This system is available with different combinations of measured parameters (Figure 6). These are zeta potential, size, $\mathrm{pH}$ and conductivity. The features of this system are its ease of use provided by pre-aligned optics and single button measurement software [9].

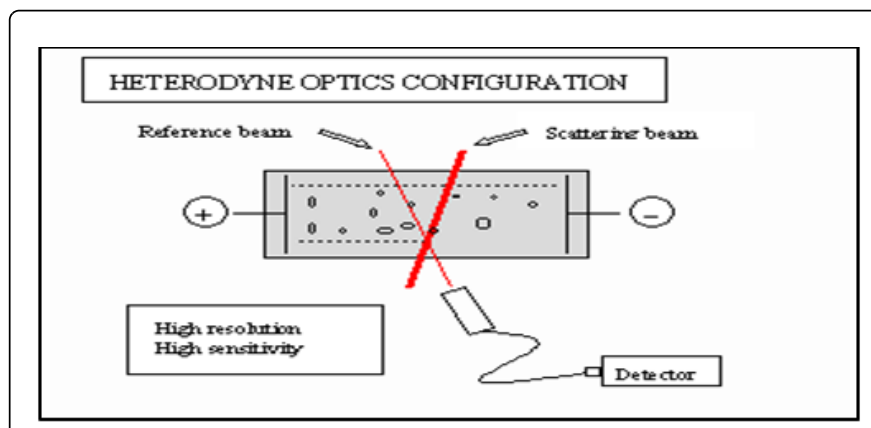

Figure 6: Measurement arrangement for the heterodyne measurement method.

\section{Heterodyne optics}

In this optical arrangement the scattered light from particles in the measurement zone is mixed with attenuated light from the illuminating laser that is directly focused on the detector. These two beams intersect at a low angle, 12.7 degrees. This effectively increases the resolution as the effect of Brownian motion is greatly reduced at forward scattering angles. At higher angles, Brownian motion causes a problem as it 'smears' out the velocity spectrum, making it difficult to resolve mixtures of particles with different mobilities [10]. 


\section{Signal processing and data evaluation}

The measurement technique used is often known as Laser Doppler Velocimetry (LDV).

Moving particles in the measurement zone shift the frequency of scattered light proportional to their velocity.

The scattered light is detected by a photon counting photomultiplier and the signal input to a digital correlator. This can be thought of as simply an efficient frequency analyzer using a Fourier transform algorithm to disentangling the mobilities of perhaps thousands or millions of particles into a number of mobility channels (Figure 7).

As well as moving due to electrophoresis, the particles are moving due to Brownian motion. This makes the peak width broader than it should appear. To correct for this a measurement is done with no field present. The width of the peak produced will then be due only to the Brownian motion, and this width can be subtracted from the width produced by a measurement with the field applied [11].

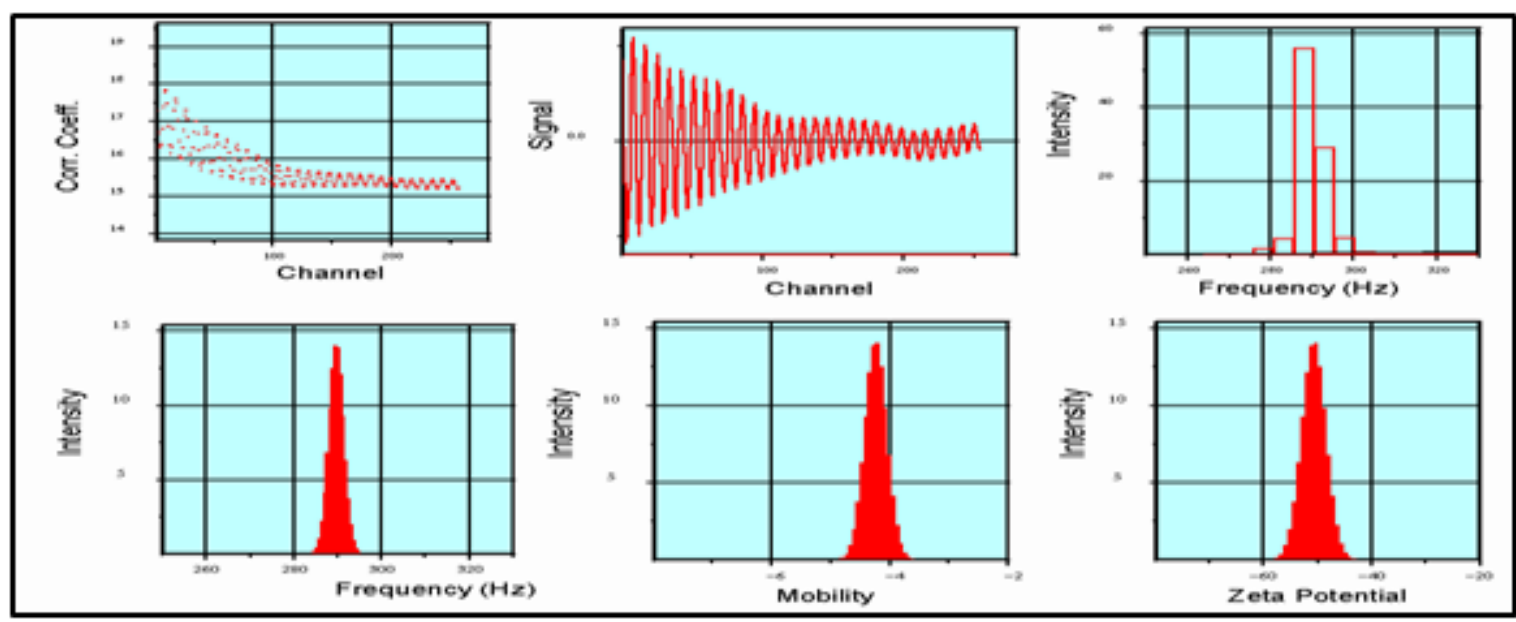

Figure 7: Stages of signal processing from the correlation function to a Fourier Transform analysis (FFT) to frequency, mobility and zeta potential spectrum.

\section{Electrode polarization and determination of sign of the zeta potential}

An alternating electrical field is used in order to exclude effects due to electrode polarization. The direction of movement of the particles is therefore periodically reversed during measurement.

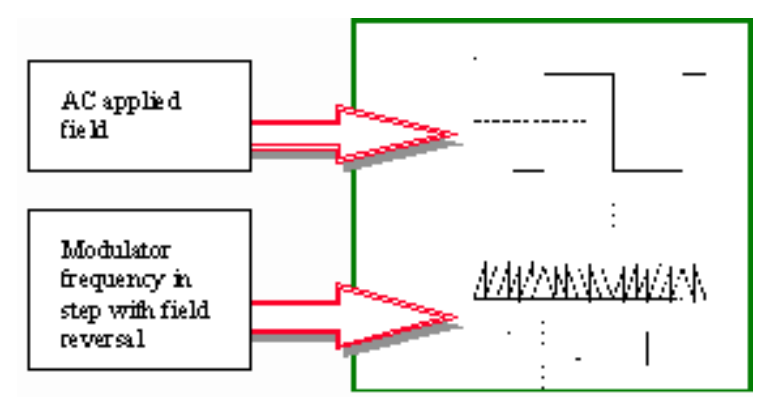

Figure 8: Link between the applied reversing field and the phase of the modulator.

In addition to this, one of the two laser beams is modulated by means of an oscillating mirror in order to determine the direction of movement and thus the sign of charge of the particle. The modulator adds a known frequency to the signal. The direction of particle movement, and therefore the sign of the charge, can be identified in this way because the frequency detected due to the particles either adds or subtracts from this modulator frequency. Figure 8 shows the time link between the direction of the electrical field and the phase of the modulated beam. With this scheme the maximum amount of data is collected with the minimum electrode polarization.

A second advantage of the modulator is that particle mobilities at zero give a measured frequency, which is that of the modulator. This means that low mobility particles can be measured with the same high accuracy as particles with high mobility.

\section{Applications - possibilities for automation}

Properties of ultrafine disperse systems (dispersions, suspensions, and emulsions) can be examined or predicted using the zeta potential. To use this parameter, it is important to understand that the zeta potential is not a parameter of a particle alone. The zeta potential is dependent, as already discussed, on the particle and on its environment. Zeta potential data with no an exact description of the conditions under which it was measured are rarely meaningful. Controlled changes of the environment, such as $\mathrm{pH}$, that lead to modification of the zeta potential, can give important information about the nature of the particle surface and the robustness of a dispersion to changes in the parameter [12].

A single measurement of the zeta potential of dispersion in a defined set of conditions is rarely useful for the purpose of characterization. Knowledge is required of how the zeta potential varies as parameters such as $\mathrm{pH}$, conductivity and other factors change 


\section{References}

1. Quin LD, Tyrell (2010) Fundamentals of Heterocyclic Chemistry. (9thedn), Wiley, New York.

2. Bruice PY (2011) Organic Chemistry (6thedn) Prentice Hall.

3. Eicher T, Hauptmann S (2003) The Chemistry of Heterocycles (2ndedn) USA: Wiley.

4. Aljamali NM (2013) Alkylation Reactions Via Organo Heteroatomes Halides. Report and Opinion 5: 33-39.

5. AlVarez-Builla J, Vaquero JJ, Barluenga J (2011) Moderen Heterocyclic Chemistry. New York: Wiley.

6. Furmeier S, Metzger J (2003) Eur J Org Chem 649-659.
7. Keniche A, MeZrai A, Mulengi JK (2011) Synthesis of a novel class of phosphonoaziridines as interesting antibacterial agents. The Open Conference proceedings J 2: 28-35.

8. Ahire JH (2011) M. Sc. Thesis, School of Chemistry, University of East Anglia, Norwich.

9. Hawraa Mahmood., M.Sc. Thesis, College of Science, University of Kufa.

10. Matsua J, Yamanaka H, Kawana A, Mukaiyama T Chemistry Letters 32: 392-393.

11. Clayden, Greeves, Warren, Wothers (2001) Organic Chemistry (1stedn), New York.

12. MaHhias D'hooghe Ir (2006) Ph.D. Thesis, Faculty of Bioscience Engineering, Ghent University. 